\title{
Study on the Technology of Combined Seal of Magnetic Fluid Seal and
}

\section{Mechanical Seal}

\author{
Shanshan Zhu' ${ }^{1,2, a}$, Xiaoxue Yang ${ }^{1, b}$, Yuting Huang ${ }^{1, c}$, Deliang Yang ${ }^{1, d}$ \\ 1 School of Mechanical and Electrical Engineering, Beijing Polytech Collage, China \\ 2 School of Mechanical and Electrical Engineering, Beijing Jiaotong University, China \\ a976148904@qq.com, b33649632@qq.com, c1361778904@qq.com, d67217840@qq.com
}

Key words: magnetic fluid seal; combined seal; pressure-resistant capacity; fluid hydrodynamic Abstract: A kind of combined seal of magnetic fluid seal and mechanical seal was designed in order to improve the capacity in the magnetic fluid seal for sealing liquid for the performance of increasing saturation magnetization and magnetic field strength was not satisfied. It mainly indicates the pressure-resistant capacity of combined seal of mechanical seal and magnetic fluid seal in this paper and verifies the pressure-resistant capacity of combined seal is better than the independent magnetic fluid seal both in the static seal and rotary seal for sealing liquid. The effect of coupling model on the performance of combined seal was studied. Results show that the film in seal end faces has large influence on some factors such as initial cone angle of the seal rings and the fluid-solid coupling effect.

\section{Introduction}

As the petrochemical, aerospace, materials science and manufacturing technology developing with high intelligence, energy saving and environmental protection, the requirements for sealing in the aerospace and other fields are increasing highly, so the modern industry puts forward some new requirements for sealing system, which are fewer leakage of sealing medium or even without leakage (including liquid phase zero leakage and gas phase zero escape) under the environment of big temperature difference and pressure difference, long service life, high stability, good anti-interference ability, low cost and convenient maintenance, etc. But the technology of traditional mechanical seal is difficult to adapt the new requirements. ${ }^{[1]}$

The seal interface usually has two basic types in all sealing forms of rotary fluid machine: one is radial seal formed by two radial located cylindrical surfaces; the other is axial seal formed by two axial located plane surfaces. ${ }^{[2]}$ The technology of traditional mechanical seal adopts the axial seal formed by seal ring end faces into rotary fluid machine, which has good anti-pressure and anti-vibration ability but has complex structure and high machining accuracy. While the magnetic fluid seal is the technology which makes the new magnetic nano-particles dispersed in base fluid to form stable colloidal solution named magnetic fluid, and then adsorbs the magnetic fluid into seal clearance under the magnetic field to form the tough annular fluid film to prevent the seal medium leaking from seal clearance. ${ }^{[3]}$ The magnetic fluid seal has zero leakage, no wear, long life and simple structure, but weak anti-temperature difference and anti-pressure difference ability. ${ }^{[4]}$ Compared with the two independent seals, the performance of combined seal of traditional mechanical seal and new magnetic fluid seal is greatly promoted.

It designs a seal structure combined traditional mechanical seal with new magnetic fluid seal, deduces and verifies that the pressure-resistant capacity of combined seal is better than that of the independent magnetic fluid seal both in the static seal and rotary seal for seal liquid, and obtains 
some results that have certain practical value. According to the elastic hydrodynamic lubrication theory, it analyzes and studies the seal mechanism of the linear groove circumferentially distributed on the seal faces. The effect that the coupling model acts on the performance of the hydrodynamic mechanical seal is further studied. The study provides a theoretical basis for the optimization of combined seal structure of mechanical seal and magnetic fluid seal and the improvement of performance.

\section{The Mechanical Seal and Magnetic Fluid Seal}

\section{The mechanical sealing principle}

The mechanical seal is also called the face seal, which is a device used to solve the seal between rotary shaft and body, and which mainly used to seal applying the elastic component to preload the seal pair in end face of rotary and static ring and to compress the medium and elastic component pressure. ${ }^{[5]}$

\section{The technology of magnetic fluid seal}

The magnetic field is a high stable colloidal solution formed by the ferromagnetism or ferromagnetic nanoparticles that are coated with surfactant dispersed highly in the base fluid of low volatility. ${ }^{[6]}$ The magnetic fluid is a new liquid magnetic function material, has the fluidity of the ordinary liquid and the magnetic conductivity of ordinary liquid which does not have. The magnetic fluid is used in sealing technology began in the 1970s, the structure is shown in Fig.1.

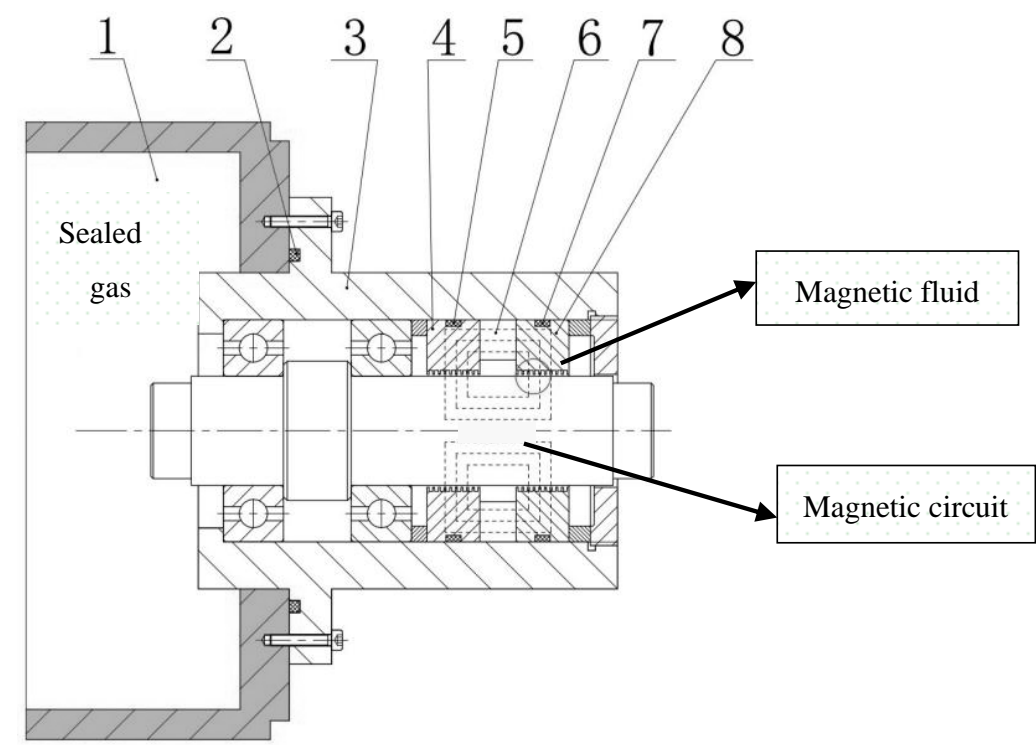

1-sealed cabin 2-seal ring 3-location sleeve 4-pole piece 5-seal ring 6-permanent magnet 7-seal ring 8-pole piece

Fig.1 The structure of magnetic fluid seal

The magnetic field is adsorbed in the seal clearance under the influence of magnetic field, which forms the tough annular fluid film to prevent the leakage of sealing medium. ${ }^{[7]}$ The magnetic particles in magnetic fluid film is separated by dispersant and base fluid and not convergent glue, which remains the liquid fluidity and without solid friction with the adsorbing parts, without wear and long service life, and its seal life mainly depends on the consumption of magnetic fluid. ${ }^{[8]}$ 


\section{The Combined Seal of Magnetic Fluid Seal and Mechanical Seal}

Based on the principle and structure of magnetic fluid seal and mechanical seal, the designed structure of combined seal of magnetic fluid seal and mechanical seal is shown in Fig.2:

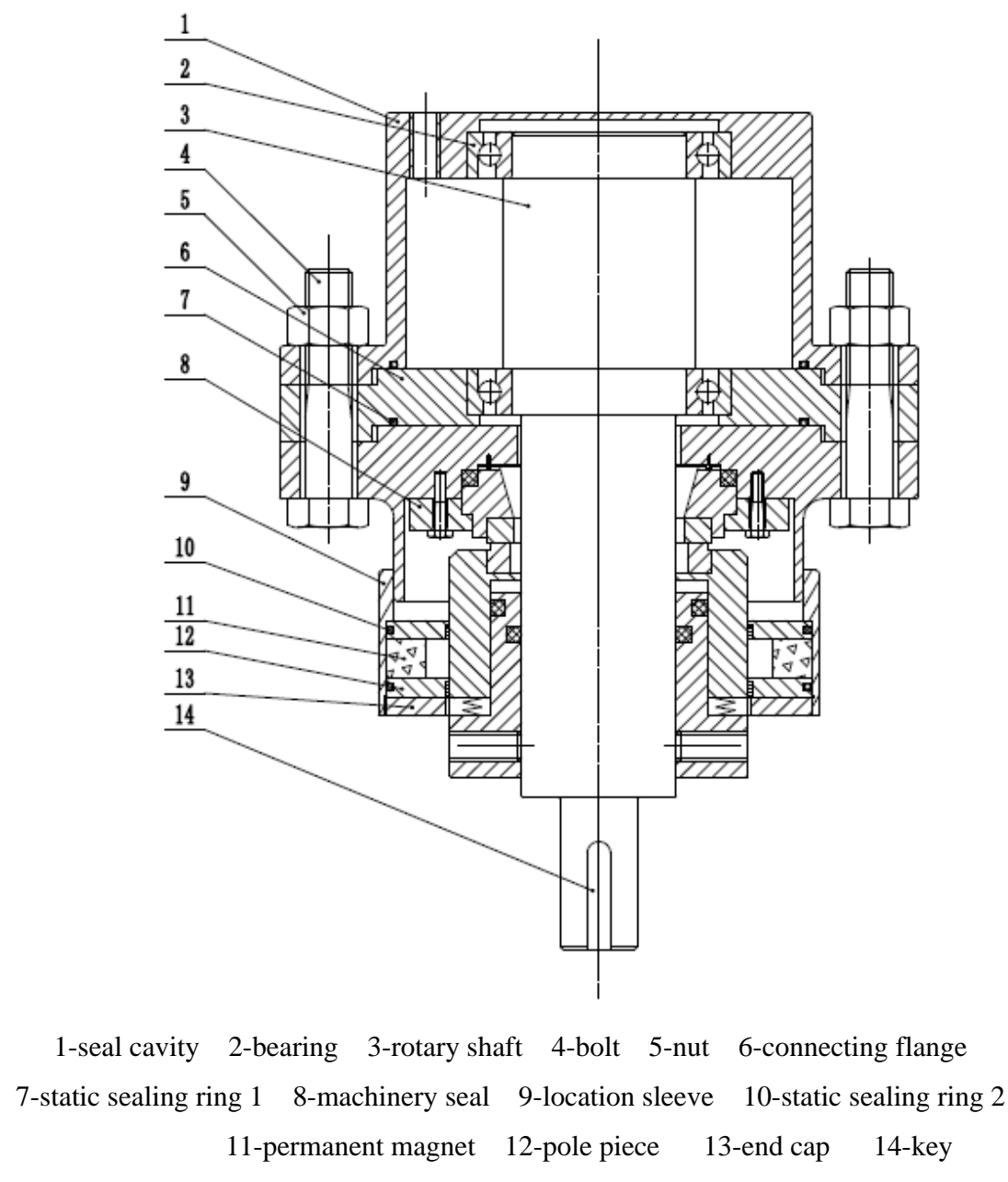

Fig. 2 The structure of combined seal with magnetic fluid seal and mechanical seal

\section{The geometrical model of combined seal}

The linear grooves are uniformly distributed along the seal ring circumference on the seal faces. Among them, the depth of linear grooves is $0.5 \mathrm{~mm}$, the width of linear grooves is $1 \mathrm{~mm}$ and the numerate of the grooves is 24, as shown in Fig.3.

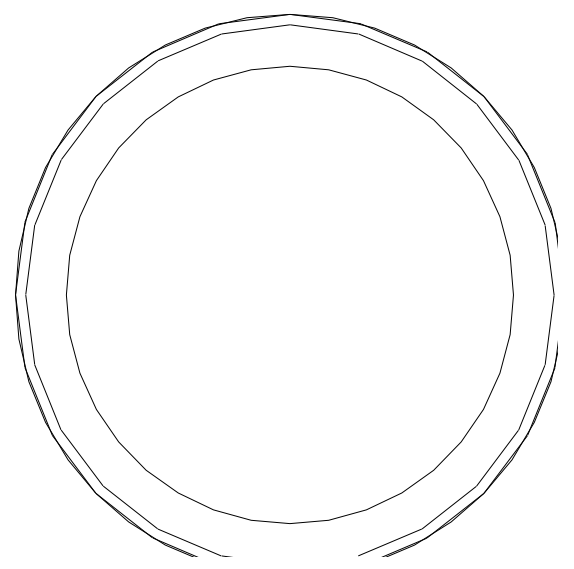

Fig.3 The end of seal ring 


\section{The mathematical model of combined seal}

To simplify the calculation, the assumption to fluid film is made as follows:

(1)The fluid film among seal faces is continuous medium and has constant temperature and viscosity;

(2)The material performance of seal ring and seal medium is constant;

(3)The seal end faces are smooth and without considering the effect of roughness on fluid film. [9]

The kinematic state of fluid film determines the seal performance of seal faces; and its control equations base on the Reynolds equation of mass conservation of polar coordinate expression:

$$
\begin{gathered}
\frac{1}{\mathrm{r}} \frac{\partial}{\partial \theta}\left(\frac{\delta^{3}}{\mathrm{r}} \frac{\partial \mathrm{p}}{\partial \theta}\right)+\frac{1}{\mathrm{r}} \frac{\partial}{\partial \mathrm{r}}\left(\mathrm{r} \delta^{3} \frac{\partial \mathrm{p}}{\partial \mathrm{r}}\right)=6 \mu \omega \frac{\partial \lambda \delta)}{\partial \theta} \\
\lambda=\frac{\rho}{\rho_{\mathrm{c}}} \quad(2)
\end{gathered}
$$

Where $\gamma$ and $\theta$ respectively represent the radius and angle in arbitrary point; $\delta$ is the thickness of fluid film; $p$ is the pressure of fluid film; $\mu$ is the viscosity of fluid film; $\omega$ is the angular speed of rotating ring; $\rho$ is the density of fluid film in arbitrary position and $\rho_{c}$ is the density of seal medium.

\section{The Fluid-solid Coupling Model}

\section{The force analysis of seal ring}

According to the axisymmetric geometry model of seal structure in combined seal, the force analysis of seal ring is shown in Fig.4.

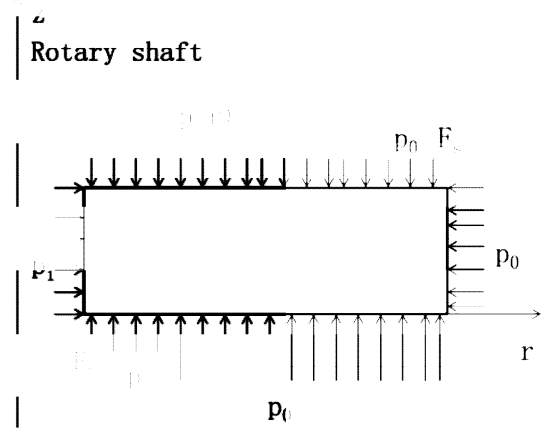

Fig.4 The force analysis of the ring

$\mathrm{p}_{0}$ is the bearing pressure along the direction of outside diameter and outside diameter side in seal faces; $p_{i}$ is the bearing pressure along the direction of inner diameter and inner diameter side in seal faces and $p(r)$ is the variation of the fluid film pressure along the radial.

\section{The deformation of the seal ring in the combined seal}

The seal rings produce mechanical deformation under load, which appears deflection around the section centroid. The comparison of deformation about the rings as shown in Fig.5. 


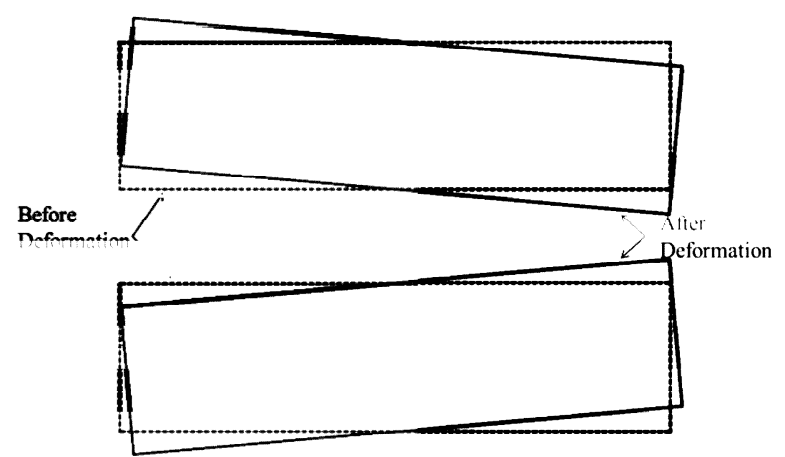

Fig. 5 The comparison of deformation about the rings

The balance equation of axial force of seal ring as follows:

$$
\mathrm{F}_{\mathrm{s}}+\mathrm{F}_{0}=\mathrm{F}_{2}+\mathrm{F}_{\mathrm{b}}
$$

$F_{s}$ - The concentration force clamping in the outside diameter of seal ring; $F_{0}-$ seal opening force; $F_{z}-$ The concentration force supporting in the inner diameter of seal ring; $F_{b}-$ seal locking force.

$F_{0}$ and $F_{b}$ can get from the following equations.

$$
\begin{aligned}
& F_{0}=2 \pi \int_{R 1}^{R 2} p(r) d r \\
& F_{b}=2 \pi \int_{R 1}^{R_{2}} p_{i} r d r
\end{aligned}
$$

There has some relationship between the deflection angle $\phi$ after the seal ring deformed and the deformation $\Delta l$ of tightening screw:

$$
\Delta \mathrm{l}=\left(\mathrm{R}_{2}-\mathrm{R}_{1}\right) \phi \quad(6)
$$

And

$$
\begin{array}{r}
\mathrm{F}_{S}=\mathrm{F}_{0}+\mathrm{k} \Delta \mathrm{l} \quad(7) \\
\phi=\frac{\mathrm{M}_{\theta} \mathrm{R}_{\mathrm{C}}^{2}}{\mathrm{EJ}{ }_{\mathrm{x}}^{2}}
\end{array}
$$

$E$ is the elastic modulus of seal ring; $\mathrm{J}_{\mathrm{X}}$ is the inertia moment of the cross section of seal ring to the $\mathrm{x}$ axis; $M_{\theta}$ is the torque produced by all of the load in the seal ring, that is

$$
\begin{aligned}
& M_{\theta}=\frac{1}{R_{c}}\left[\int_{R_{1}}^{R_{2}} p_{i}\left(r-R_{c}\right) r d r-\int_{R_{1}}^{R_{2}} p\left(r-R_{c}\right) r d r\right]+ \\
& \frac{1}{R_{c}}\left[\int_{0}^{Z_{2}} p_{0}\left(z-Z_{c}\right) R_{2} d z-\int_{0}^{z_{2}} p_{i}\left(z-Z_{c}\right) R_{1} d z\right]+ \\
& \frac{1}{2 \pi R_{c}}\left[F_{z}\left(R_{1}-R_{c}\right)-F_{s}\left(R_{2}-R_{c}\right)\right]
\end{aligned}
$$


Also $\quad J_{x}=\int_{A} y^{2} /\left(1-x / R_{c}\right) d A$

Where $R_{1}$ and $R_{2}$ respectively represent the inner diameter and outside diameter in the seal ring;

$\mathrm{C}$ is the centroid of the cross section of seal ring; $R_{c}$ is the radius of the centroid $\mathrm{C}$; $\mathrm{Z}_{1}$ and $Z_{2}$ respectively represent the $\mathrm{Z}$ coordinate of low and top angle point in the outside of seal ring. Therefore, the deformation of seal ring can be obtained.

\section{The analysis of the flow field in seal faces}

In axisymmetric model, the control equations of fluid film in the seal clearance are as follows:

$$
\begin{aligned}
& \frac{\partial}{\partial r}\left(r \delta^{3} \frac{\partial p}{\partial r}\right)=0 \\
& \left.p\right|_{r=R_{1}}=p_{i} \\
& \left.p\right|_{r=R_{2}}=p_{0}
\end{aligned}
$$

Then $p(r)=\frac{p_{0}\left(f(r)-f\left(R_{1}\right)\right)-p_{i}\left(f(r)-f\left(R_{1}\right)\right)}{f\left(R_{2}\right)-f\left(R_{1}\right)}$

$$
f(r)=\int \frac{1}{r^{3}{ }^{3}} d r
$$

One can get

$$
\begin{array}{r}
\frac{d p}{d r}=\frac{1}{r \delta^{3}} \frac{p_{0}-p_{i}}{\zeta} \\
\zeta\left(\delta_{0}, \phi\right)=\int_{R_{1}}^{R_{2}} \frac{1}{r \delta^{3}} d r
\end{array}
$$

The $\delta_{0}$ is the minimum film thickness in the equilibrium state.

According to the above equations, the leakage $\mathrm{Q}$ and the opening force $F_{0}$ can be obtained.

$$
\begin{gathered}
Q=2 \pi R \frac{\delta^{3}}{12 \mu} \frac{d p}{d r}=\frac{\pi\left(p_{0}-p_{i}\right)}{6 \mu \zeta} \\
F_{0}=2 \pi \int_{R_{1}}^{R_{2}} p r d r=2 \pi\left(\left.\frac{p r^{2}}{2}\right|_{R_{1}} ^{R_{2}}-\int_{P_{i}}^{P_{0}} \frac{r^{2}}{2} d p\right)=\pi\left(p_{0} R_{2}^{2}-p_{1} R_{1}^{2}\right)-\pi\left(p_{0}-p_{i}\right) \frac{\eta}{\zeta} \\
\eta\left(\delta_{0}, \phi\right)=\int_{R_{1}}^{R_{2}} \frac{r}{\delta^{3}} d r
\end{gathered}
$$

$\mu$ is the kinematic viscosity of seal medium. 


\section{The experiment of the combined sealing liquid of magnetic fluid seal and mechanical seal}

\section{The static sealing liquid experiment of combined seal}

Before this experiment started, seal clearance is injected into $5 \mathrm{ml}$ magnetic fluid, combined seal structure is installed in test bed, and inlet valve and pressure gauge are connected. This experiment uses water as liquid sealing medium.

The sealed cabin is pressurized after injected into $20 \mathrm{ml}$ water. From the minimum pressure value $0.05 \mathrm{MPa}$ starting, the sealed cabin is pressurized $0.05 \mathrm{MPa}$ every other hour until the pressure reaches $0.5 \mathrm{MPa}$. Through observing the pressure gauge readings and recording readings before pressurizing every time, the experimental data of static seal for water by combined seal and magnetic fluid seal is shown in Fig.6.

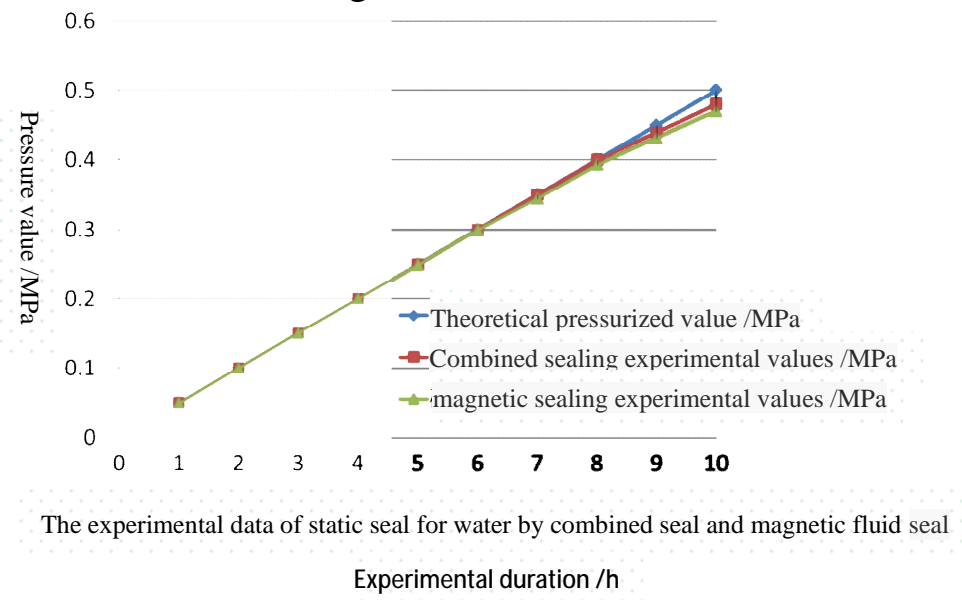

Fig.6 The experimental data of static seal for water by combined seal

\section{The dynamic sealing liquid experiment of combined seal}

The connection performance of all components is debugged before the experiment. The sealed cabin is injected into $20 \mathrm{ml}$ water, which the experiment uses the experimental method of static seal for water by combined seal to monitor the seal anti-pressure performance of dynamic seal for water by combined seal respectively in the speed at 200 $/ \mathrm{min}, 250 \mathrm{r} / \mathrm{min}$ and $300 \mathrm{r} / \mathrm{min}$, observes the experimental phenomenon and record the pressure gauge readings, the anti-pressure experimental data of static seal for water by combined seal and magnetic fluid is shown in Fig.7(a), 7(b).
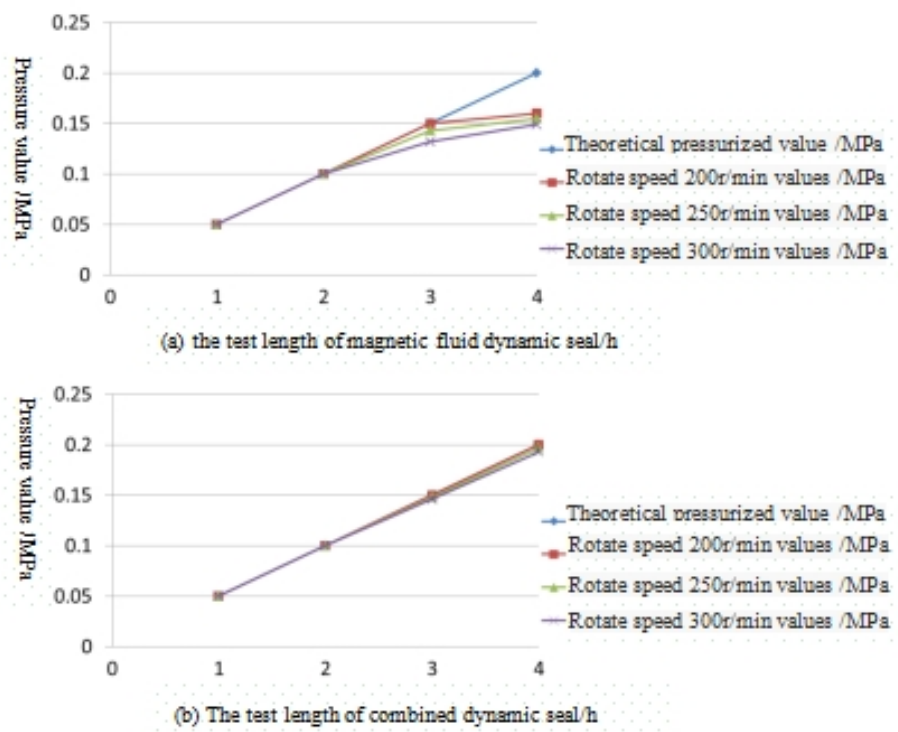

Fig.7 The experimental data of dynamic seal for water by combined seal 


\section{The Finite Element Analysis of Combined Seal}

The ANSYS finite element method is used to verify the analytical method of the mechanical deformation of seal ring from the moment when the deflection angle $\phi$ of seal ring is equal to zero and the $\delta_{0}$ in the equilibrium state is obtained in the fluid-solid coupling model. Then the situations of stress distribution and deformation of seal ring according to the ANSYS finite element method are respectively shown in Fig.8 and Fig.9.

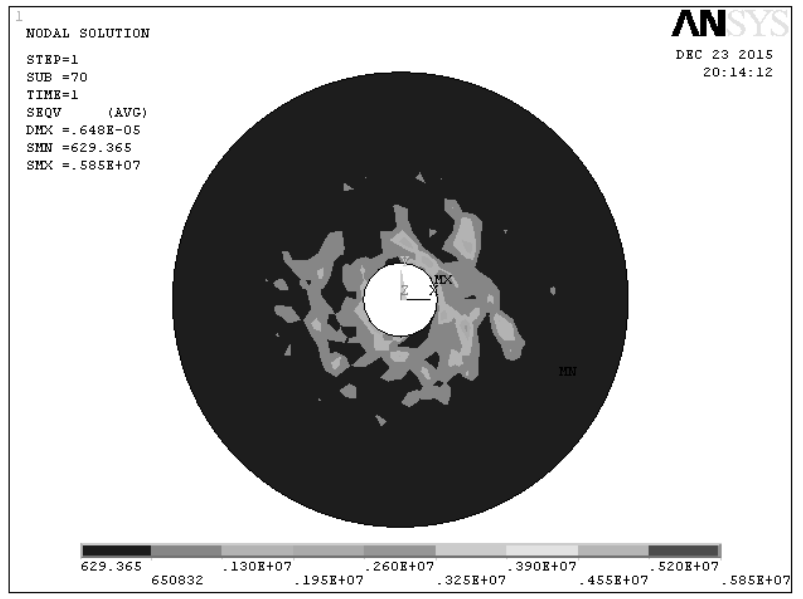

Fig.8 Stress distribution of seal ring

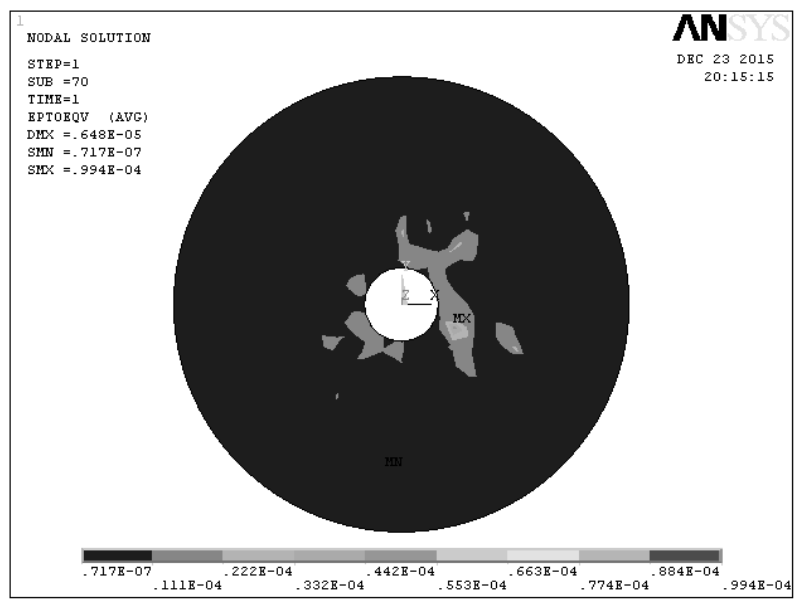

Fig.9 Deformation of seal ring

The circumferential waviness in the end faces is formed by the double effect on the fluid film of seal faces and the pressure of seal medium; and the taper of lubricant film also increases with the increasing radial deformation of seal ring.

\section{Conclusions}

(1) By the anti-pressure experiment of static seal for water of Combined Seal, the anti-pressure performance of static sealing liquid of combined seal is higher than that of independent magnetic fluid seal, which the combined seal can reach $0.45 \mathrm{Mpa}$. The combined seal of magnetic fluid seal and mechanical seal is a critical way for improving the capacity of magnetic fluid sealing anti-pressure.

(2) By the anti-pressure experiment of dynamic seal for water of combined seal, the seal performance can reach $0.2 \mathrm{MPa}$ that is superior to independent magnetic liquid rotary seal when the rotary speed at 200r/min. The combined seal of magnetic fluid seal and mechanical seal is a 
technology that uses the magnetic fluid seal in seal fluid medium and is an important breakthrough to solve the problem of some critical technologies.

(3)Through analyzing the fluid-solid coupling model's influence to the combined seal performance, the results are that the mechanical deformation in seal faces has great influence on the lubrication film in the seal gap and the initial cone angle in seal faces should be combined with fluid-solid coupling interaction to comprehensively analyze the impact of the combined seal performance when studying numerical calculation of seal performance.

(4) It is found that the seal faces of rotating rings and static rings can form the circumferential waviness and the radial taper along the seal ring under the effect of linear grooves in seal faces. When the pressure elevates, the circumferential waviness becomes larger while the radial taper is fewer changed, which reduces the leakage rate to some extent and provides a theoretical basis for the optimization of combined seal structure of mechanical seal and magnetic fluid seal and the improvement of performance.

\section{Acknowledgments}

This research was supported by the General Science and Technology Project of Beijing Municipal Education Commission (Grant No. KM201610853001).

\section{References}

[1]Djamai A, Brunetiere N, Tournerie B. Numerical modeling of thermo hydrodynamic mechanical faces seals [J]. Tribology Transactions, 2010, 53(3) : 414-425.

[2]Ausas R F, Jai M, Buscaglia G C. A mass -conserving algorithm for dynamical lubrication problems with cavitation[J]. Journal of Tribology, 2009, 131( 3), 1-17.

[3]Liao C J, Huang W F, Suo S F, et al. Semi-analytical fluid-solid coupling model for hydrostatic mechanical seals [J]. Journal of Mechanical Engineering, 2010, 46 (20):145-151.

[4]Loenhout G V, Olson A and J, Olliver, et al. Improving reactor coolant pump seal reliability at Dominion Surry nuclear power station [C]. Fluid Sealing Conference, Milton Keynes, 2011.

[5] LI Decai, YAO Jie. Peltier cooling mode magnetic fluid sealing device : China , 201410177531.8[P].2014. (in Chinese)

[6] CHI Changqing, WANG Zhishan, ZHAO Pizhi. Ferrohydrodynamics[M]. Beijing: Beihang University Press, 1993:181-182.

[7]ERNEL C S, ERDEM K. An investigation on the lubrication mechanisms of the mechanical radial face seals-I: General theory[J]. Industrial Lubrication and Tribology, 2007, 59 (2):85-91.

[8]SUN Jianjun. Prediction theory on leakage of mechanical seal and application[M]. Beijing: China Electric Power Press, 2011.

[9] XU Hao. Seal[M]. Beijing: Metallurgical Industry Press, 1999. 